\title{
Update of the ALEPH non-strange spectral functions from hadronic $\tau$ decays
}

\author{
M. Davier ${ }^{1}$, A. Höcker ${ }^{2}$, B. Malaescu ${ }^{3}$, C. Z. Yuan ${ }^{4}$, Z. Zhang $^{1, \mathrm{a}}$ \\ ${ }^{1}$ Laboratoire de l'Accélérateur Linéaire, IN2P3-CNRS et Université Paris-Sud 11, 91898 Orsay Cedex, France \\ ${ }^{2}$ CERN, 1211 Geneva 23, Switzerland \\ ${ }^{3}$ Laboratoire de Physique Nucléaire et des Hautes Energies, IN2P3-CNRS et Universités Pierre-et-Marie-Curie et Denis-Diderot, 75252 Paris \\ Cedex 05, France \\ ${ }^{4}$ Institute of High Energy Physics, Chinese Academy of Sciences, Beijing, China
}

Received: 4 January 2014 / Accepted: 26 February 2014 / Published online: 15 March 2014

(c) The Author(s) 2014. This article is published with open access at Springerlink.com

\begin{abstract}
An update of the ALEPH non-strange spectral functions from hadronic $\tau$ decays is presented. Compared to the 2005 ALEPH publication, the main improvement is related to the use of a new method to unfold the measured mass spectra from detector effects. This procedure also corrects a previous problem in the correlations between the unfolded mass bins. Results from QCD studies and for the evaluation of the hadronic vacuum polarisation contribution to the anomalous muon magnetic moment are derived using the new spectral functions. They are found in agreement with published results based on the previous set of spectral functions.
\end{abstract}

\section{Introduction}

Because of its relatively large mass and the simplicity of its decay mechanism, the $\tau$ lepton offers many interesting and sometimes unique possibilities for testing the Standard Model. Among these, the production of hadrons from the QCD vacuum has been widely studied. The $\tau$ data were proved to be complementary to data from $e^{+} e^{-}$annihilation, allowing one to perform detailed studies at the fundamental level through the determination of the spectral functions, which embody both the rich hadronic structure seen at low energy, and the quark behaviour relevant at higher energy. The spectral functions play an important role in the understanding of hadron dynamics at intermediate energies and they form a basic ingredient in QCD studies and in evaluating hadronic vacuum polarisation effects. Robust predictions of these effects are needed for precision tests of electroweak theory through the running of $\alpha$ to the $M_{\mathrm{Z}}$ scale, and to compute the anomalous magnetic moment of the muon. For the

a e-mail: zhangzq@1al.in2p3.fr latter application the spectral function of the $\pi \pi^{0}$ state is of paramount importance as it dominates the hadronic vacuum polarisation contribution.

Following earlier determinations $[1,2]$ the ALEPH Collaboration released results in 2005 on the $\tau$ branching fractions and spectral functions based on the complete available data statistics [3]. The spectral function data and their covariance matrix were made public and they have been used in many phenomenological studies. A problem with the covariance matrix became apparent when fits to the spectral functions were performed [4]. The statistical bin-to-bin correlations introduced by the unfolding procedure were not included.

After a short introduction in Sect. 2, we present in Sects. 3 and 4 the updated spectral functions based on unchanged reconstructed data, but correcting the unfolding procedure to obtain a complete covariance matrix that properly includes all bin-to-bin correlations. We also utilised a new, more robust unfolding method [5]. A fit of the $\rho$ line shape to the unfolded $\pi \pi^{0}$ data is presented in Sect. 5. We repeat in Sect. 6 the QCD studies performed previously [3], and present in Sect. 7 an updated evaluation of hadronic vacuum polarisation contributions to the muon magnetic anomaly using the $\tau$ spectral functions from ALEPH and other experiments. Both the QCD and hadronic vacuum polarisation results are found in agreement with those published in [3].

\section{Spectral functions from non-strange hadronic $\tau$ decays}

The definition and determination of spectral functions are described in detail in Ref. [6], and only a few generalities are recalled here. The spectral function $v_{1}\left(a_{1}, a_{0}\right)$, where the 
subscript refers to the spin $J$ of the hadronic system, is defined for a non-strange $(|\Delta S|=0)$ vector (axial-vector) hadronic $\tau$ decay channel $V^{-} v_{\tau}\left(A^{-} v_{\tau}\right)$. The spectral function is obtained by dividing the normalised invariant mass-squared distribution $\left(1 / N_{V / A}\right)\left(d N_{V / A} / d s\right)$ for a given hadronic mass $\sqrt{s}$ by the appropriate kinematic factor

$$
\begin{aligned}
& v_{1}(s) / a_{1}(s)=\frac{m_{\tau}^{2}}{6\left|V_{u d}\right|^{2} S_{\mathrm{EW}}} \frac{B\left(\tau^{-} \rightarrow V^{-} / A^{-} v_{\tau}\right)}{B\left(\tau^{-} \rightarrow e^{-} \overline{\nu_{e}} \nu_{\tau}\right)} \frac{d N_{V / A}}{N_{V / A} d s} \\
& \quad \times\left[\left(1-\frac{s}{m_{\tau}^{2}}\right)^{2}\left(1+\frac{2 s}{m_{\tau}^{2}}\right)\right]^{-1} \\
& a_{0}(s)=\frac{m_{\tau}^{2}}{6\left|V_{u d}\right|^{2} S_{\mathrm{EW}}} \frac{B\left(\tau^{-} \rightarrow \pi^{-} v_{\tau}\right)}{B\left(\tau^{-} \rightarrow e^{-} \overline{v_{e}} v_{\tau}\right)} \frac{d N_{A}}{N_{A} d s} \\
& \quad \times\left(1-\frac{s}{m_{\tau}^{2}}\right)^{-2}
\end{aligned}
$$

where $S_{\mathrm{EW}}$ accounts for short-distance electroweak radiative corrections. Since isospin symmetry is a very good approximation for the non-strange sector, the $J=0$ contribution to the non-strange vector spectral function is put to zero, while the main contributions to $a_{0}$ are from the pion pole, with $d N_{A} / N_{A} d s=\delta\left(s-m_{\pi}^{2}\right)$. The spectral functions are normalised by the ratio of the vector/axial-vector branching fraction $B\left(\tau^{-} \rightarrow V^{-} / A^{-} v_{\tau}\right)$ to the branching fraction of the massless leptonic, i.e., electron, channel. The CKM matrix element $\left|V_{u d}\right|=0.97418 \pm 0.00019$ is taken from Ref. [7].

The measurement of the $\tau$ spectral functions defined in Eq. (1) requires the determination of the invariant masssquared distributions, obtained from the experimental distributions after correcting for the effects of measurement distortion. The unfolding procedure used by the ALEPH collaboration, initially [1,2] and in Ref. [3], was based on the regularised inversion of the simulated detector response matrix using the Singular Value Decomposition (SVD) technique [8]. The regularisation function applied minimised the average curvature of the distribution and the optimal choice of the regularisation strength was found by means of the Monte Carlo (MC) simulation where the true distribution was known.

Before unfolding the mass distributions, the $\tau$ and non- $\tau$ backgrounds are subtracted. In the case of $\tau$ feed-through the MC distributions normalised to the measured branching fractions from ALEPH [3] are used. The contributions from strange modes classified in the same topology are subtracted using their MC spectral functions normalised by the measured branching fractions.

The systematic uncertainties affecting the decay classification of the exclusive modes are contained in the systematic errors of the measured branching fractions. Additional systematic uncertainties related to the shape of the unfolded mass-squared distributions, and not its normalisation, are also included. They are dominated by the photon and $\pi^{0}$ reconstruction.

\section{Update of the analysis using a new unfolding method}

The unfolding technique used in this reanalysis is a simplified version of a method developed for more complex unfolding problems [5]. The folding probability $P_{i j}$ of an event produced in a true mass bin $j$ to be reconstructed in a mass bin $i$ is computed directly in MC simulation from the transfer matrix $A_{i j}$ (the number of events produced in a true bin $j$ that are reconstructed in bin $i) .{ }^{1}$ Conversely, the matrix of unfolding probabilities $P_{i j}^{\prime}$ indicates the probability for an event reconstructed in a bin $i$ to originate from the true bin $j$, and is also computed from the transfer matrix. $A_{i j}$ and $P_{i j}^{\prime}$ depend on the assumed true spectrum while $P_{i j}$, which describes detector and final state radiation effects, to good approximation does not. The method used to unfold the mass spectra is based on the idea that if the MC describes well enough the true spectrum in data and if the folding probabilities are well simulated, the matrix of unfolding probabilities determined in simulation can be applied to data.

If the first condition is not fulfilled, that is if the data spectrum after unfolding differs significantly from the true MC spectrum, several steps are iterated in which the transfer matrix is improved by re-weighting the true MC, keeping the folding probabilities unchanged. Differences between data and folded ('reconstructed') MC spectra are ascribed to differences in the unfolded ('true') spectra. At each step of the iterative re-weighting process, the data-MC differences of the reconstructed spectra are unfolded and added to the true MC spectrum. Such iterative procedures can result in a significant bias in the final results if statistical fluctuations are mis-interpreted as genuine differences between data and MC distributions. The method is therefore stabilised with the use of a regularisation function that suppresses large fluctuations in the unfolded data. The new unfolding method is using a weaker regularisation (based on the significance of the dataMC differences in each bin of the spectrum) than the SVD approach which imposes constraints on the average curvature of the spectrum [8]. Therefore, the new method induces less smoothing and correlations between mass bins. Details on the method are given in [5].

It is important to ensure that the MC simulation correctly reproduces the calibration and the resolution of the observed hadronic mass, which are dominated by the $\pi^{0} /$ photon measurement. Specific studies are performed using electrons from $\tau$ decays as a function of energy, and corrections are

\footnotetext{
${ }^{1}$ The matrix of folding probabilities is related to the transfer matrix $A_{i j}$ by $P_{i j}=A_{i j} / \sum_{k=1}^{N} A_{k j}$ while the matrix of unfolding probabilities is $P_{i j}^{\prime}=A_{i j} / \sum_{k=1}^{N} A_{i k}$.
} 
Fig. 1 Left relative difference between data (d) and reconstructed $\mathrm{MC}$ spectrum before unfolding (rMC) and after 5 (rMCm5) and 6 (rMCm6) iterations for the $\pi \pi^{0}$ channel. Right relative difference either between unfolded spectrum and the data or between unfolded spectra after 1 (UR1), 6 (UR6) and 7 (UR7) iterations for the same channel

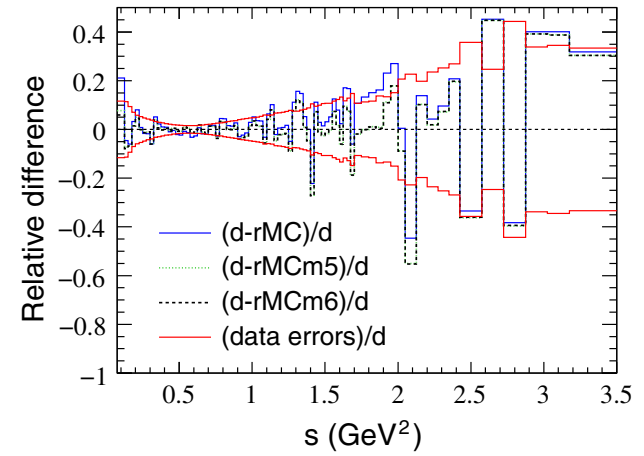

applied to the simulation to match the properties of the data. The systematic uncertainties have been revisited following these studies.

Different numbers of bins with varying bin sizes are chosen for the unfolding depending on the available statistics. These are 83, 97, 29, 91 and 96 for the $\pi \pi^{0}, \pi 2 \pi^{0}, \pi 3 \pi^{0}$, $3 \pi$ and $3 \pi \pi^{0}$ channels, respectively. For the vector and axialvector spectra, obtained by summing the appropriate channels, a common number of 80 bins is adopted. The same mass-squared range up to $3.5 \mathrm{GeV}^{2}$ is used.

The left plot in Fig. 1 shows the agreement between data and reconstructed MC for the $\pi \pi^{0}$ channel for different numbers of iterations. The agreement improves with each iteration, reaching a satisfactory level after five iterations, the impact of further steps being very small. The right plot in Fig. 1 displays the relative correction to the measured spectrum resulting from the unfolding. Most of the correction is applied with the first iteration step, namely (UR1-data).

A data-driven closure test is performed to optimise the number of iterations and to evaluate the systematic uncertainty due to the unfolding method. To achieve this the true MC spectrum is reweighted using a smooth function to improve the agreement between data and the reconstructed MC. The so reweighted MC spectrum is then reconstructed and provided as input to the unfolding process that uses the same transfer matrix as for data. The comparison between the reweighted true MC spectrum and the unfolded one provides a measure of the bias introduced by the method. The results are shown in Fig. 2 for the $\pi \pi^{0}$ channel. After 5 iterations the relative difference is very small and negligible compared to the other sources of systematic uncertainties. Inserting additional statistical fluctuations in the closure test to decorrelate the $\mathrm{MC}$ events used in the unfolding from those entering the response matrix does not noticeably alter the result.

\section{Results}

A comparison of the new unfolded mass spectra with the previous ones [3] is shown in Fig. 3. Reasonable agreement is found everywhere except for differences at the few percent

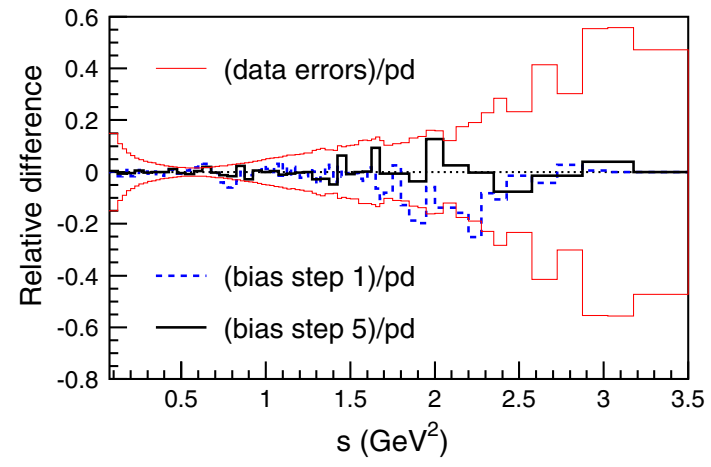

Fig. 2 Results from the unfolding closure test using pseudodata (pd) for the $\pi \pi^{0}$ channel. The histograms show the relative difference (bias) between the true MC spectrum and the result after unfolding of the reconstructed distribution in two cases: after the first iteration (dashed) and after five iterations (solid bold). For comparison the relative statistical uncertainty on the pseudodata spectrum is indicated by the band between the two light histograms. The fluctuations in the unfolded spectrum after five iterations are of mainly statistical origin. Rebinning to broader bins reveals a negligible systematic bias

level in the $\pi \pi^{0}$ mode near threshold and in the $0.8-1.0 \mathrm{GeV}^{2}$ region. One also observes some structures in the $3 \pi \pi^{0}$ mode which was not present in the previous analysis. In fact such a structure was already there in the raw mass spectrum, but was smoothed away by our implementation of the SVD unfolding. An increased statistical uncertainty is also observed near the edges of phase space due to the reduced regularisation in the unfolding method employed here.

Following the procedure defined in Ref. [9], the updated ALEPH $\pi \pi^{0}$ spectral function is combined with the published results from CLEO [10], OPAL [11] and Belle [12]. The relative comparison of the individual spectral functions with the combination is shown in Fig. 4. It is in good agreement with a similar comparison based on the previous ALEPH spectral functions [9]. In particular, the tension above $0.85 \mathrm{GeV}^{2}$ between ALEPH, CLEO, and OPAL on one side and Belle on the other side still persists, although it is somewhat reduced with the new ALEPH unfolding.

A spectacular dip was found by Belle [12] near $2.4 \mathrm{GeV}^{2}$ and confirmed in the $e^{+} e^{-} \rightarrow \pi^{+} \pi^{-}$cross section by BABAR $[13,14]$. As before the much lower statistics of the 
Fig. 3 Comparison of the new unfolded spectral functions (red full circles) with those obtained in Ref. [3] (black open circles, denoted "Old"). The error bars shown include statistical and all systematic uncertainties. The insets show the old-to-new ratios for better visibility, where the error bars are those of the newly unfolded spectra. For the $\pi 3 \pi^{0}$ and $3 \pi \pi^{0}$ channels the spectra are directly compared near the peak since new and previous data are not given in the same energy bins
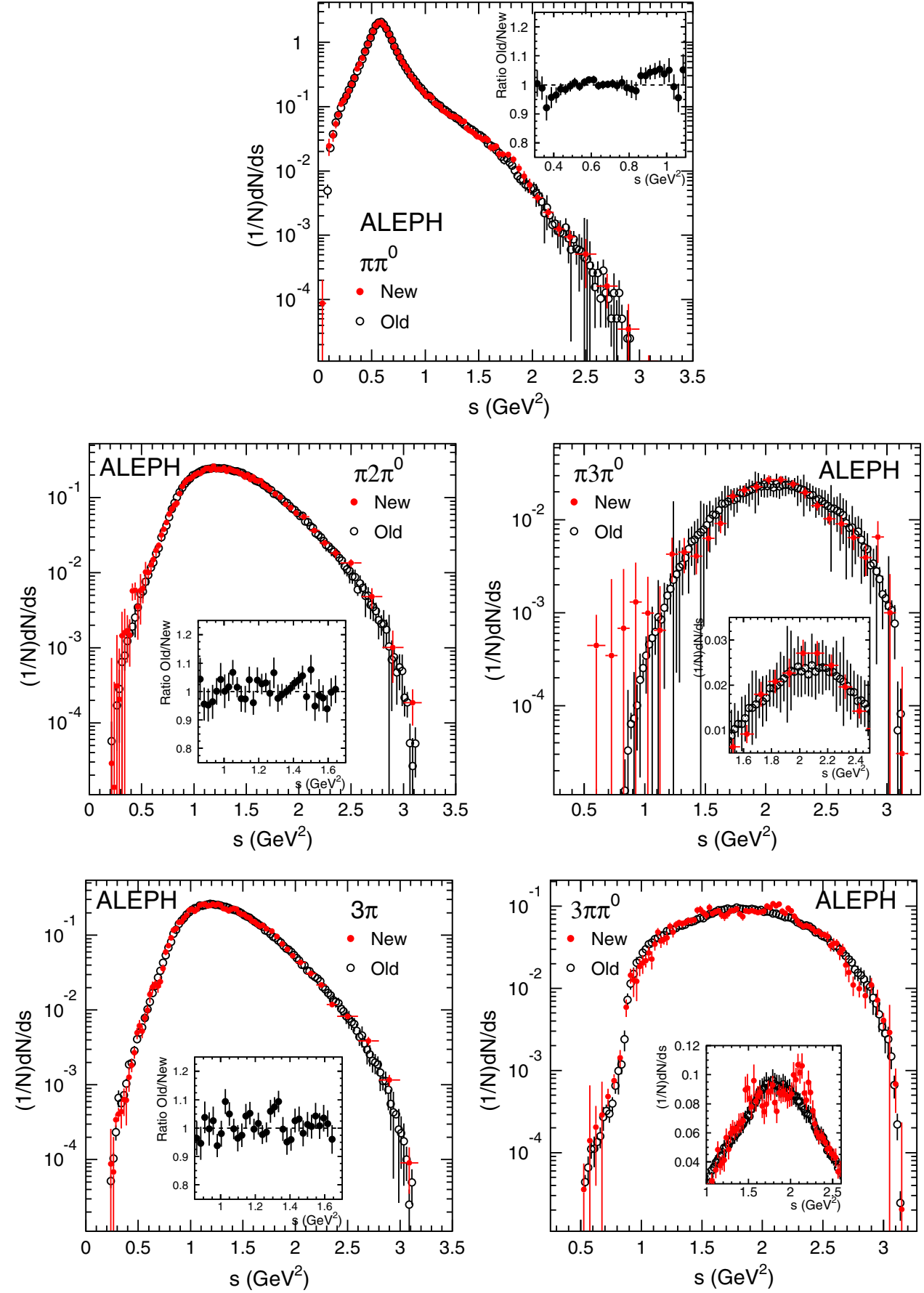

ALEPH data (and similarly for CLEO and OPAL) does not permit to resolve this structure.

The new vector $(V)$, axial-vector $(A), V+A$ and $V-A$ spectral functions are displayed in Fig. 5. The correlation matrices, shown in Fig. 6 for the vector part, have been carefully checked using pseudodata. Data for the updated spectral functions and their correlation matrices are publicly available [15].

\section{The $\rho$ line shape in the $\pi \pi^{0}$ channel}

The $\pi \pi^{0}$ spectral function is dominated by the wide $\rho$ resonance that can be parametrised following Gounaris-Sakurai (GS) [16]. The statistical estimator minimised in the fit accounts for the correlations between different mass bins.

If one assumes vector dominance, the pion form factor is given by interfering amplitudes from the known isovector 

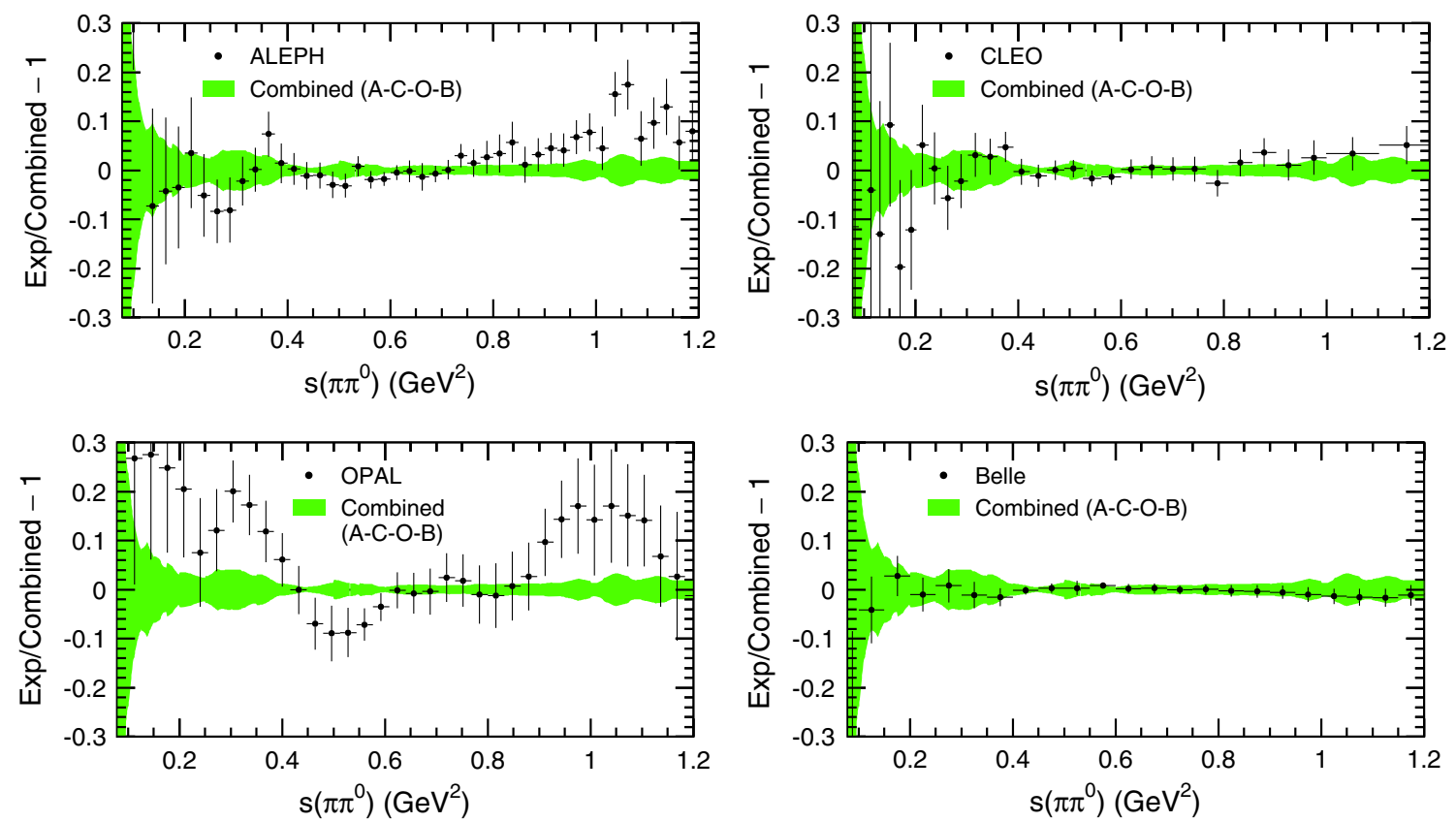

Fig. 4 Relative comparison between the $\tau^{-} \rightarrow \pi^{-} \pi^{0} v_{\tau}$ invariant mass-squared measurements from ALEPH, CLEO, OPAL and Belle (data points) and the new combined result (shaded band). This figure supersedes Fig. 1 of [9]

meson resonances $\rho$ (770), $\rho(1450)$ and $\rho(1700)$ with relative strengths $1, \beta$, and $\gamma$. Although one could expect from the quark model that $\beta$ and $\gamma$ are real and respectively negative and positive, the phase of $\beta, \phi_{\beta}$ is left free in the fits, while the much smaller parameter $\gamma$ is assumed to be real for lack of precise experimental information at large mass.

The parametrisation used can be found in the previous ALEPH paper [3]. The fitted resonance parameters given in Table 1 are in good agreement with those obtained with the previous $\pi \pi^{0}$ spectral function, except for the $\rho(770)$ width which comes out larger here. The uncertainties of the fitted quantities are increased with the re-evaluation of the systematic uncertainties on the mass calibration and resolution, and the new covariance matrix.

\section{Update of the QCD analysis}

We update the QCD analysis of Ref. [3] (and references therein) with the new spectral functions. Here we follow the same notations and only briefly recall our method. A simultaneous fit of QCD predictions is performed including perturbative and nonperturbative components to the measured ratio $R_{\tau}$

$R_{\tau}=\frac{\Gamma\left(\tau^{-} \rightarrow \text { hadrons }^{-} v_{\tau}\right)}{\Gamma\left(\tau^{-} \rightarrow e^{-} \bar{v}_{e} v_{\tau}\right)}$

and to the spectral moments defined by
$R_{\tau, V / A}^{k l} \equiv \int_{0}^{m_{\tau}^{2}} d s\left(1-\frac{s}{m_{\tau}^{2}}\right)^{k}\left(\frac{s}{m_{\tau}^{2}}\right)^{l} \frac{d R_{\tau, V / A}}{d s}$,

with $R_{\tau, V / A}^{00}=R_{\tau, V / A}$. The values for $R_{\tau, V}=1.782 \pm$ $0.009, R_{\tau, A}=1.694 \pm 0.010, R_{\tau, V+A}=3.475 \pm 0.011$, determined by the respective branching fractions, are updated with very small changes from Ref. [17]. Note that the $V+$ $A$ branching fraction is obtained as one minus the sum of leptonic and strange branching fractions.

For practical purpose, normalised moments decorrelating normalisation and shape information between $R_{\tau}$ and the spectral moments are used

$D_{\tau, V / A}^{k l} \equiv \frac{R_{\tau, V / A}^{k l}}{R_{\tau, V / A}}$.

Their experimental values are given in Table 2 and their correlation matrices in Table 3. While the central values are in agreement with those from Ref. [3], somewhat larger correlations between $R_{\tau, V / A}$ and $D_{\tau, V / A}^{10}$, and smaller correlations between higher moments, are observed here.

The theoretical prediction of the vector and axial-vector ratio $R_{\tau, V / A}$ can be written as (see references and details in $[3,6])$ :

$$
\begin{aligned}
& R_{\tau, V / A}=\frac{3}{2}\left|V_{u d}\right|^{2} S_{\mathrm{EW}} \\
& \times\left(1+\delta^{(0)}+\delta_{\mathrm{EW}}^{\prime}+\delta_{u d, V / A}^{(2-\text { mass })}+\sum_{D=4,6, \ldots} \delta_{u d, V / A}^{(D)}\right),
\end{aligned}
$$



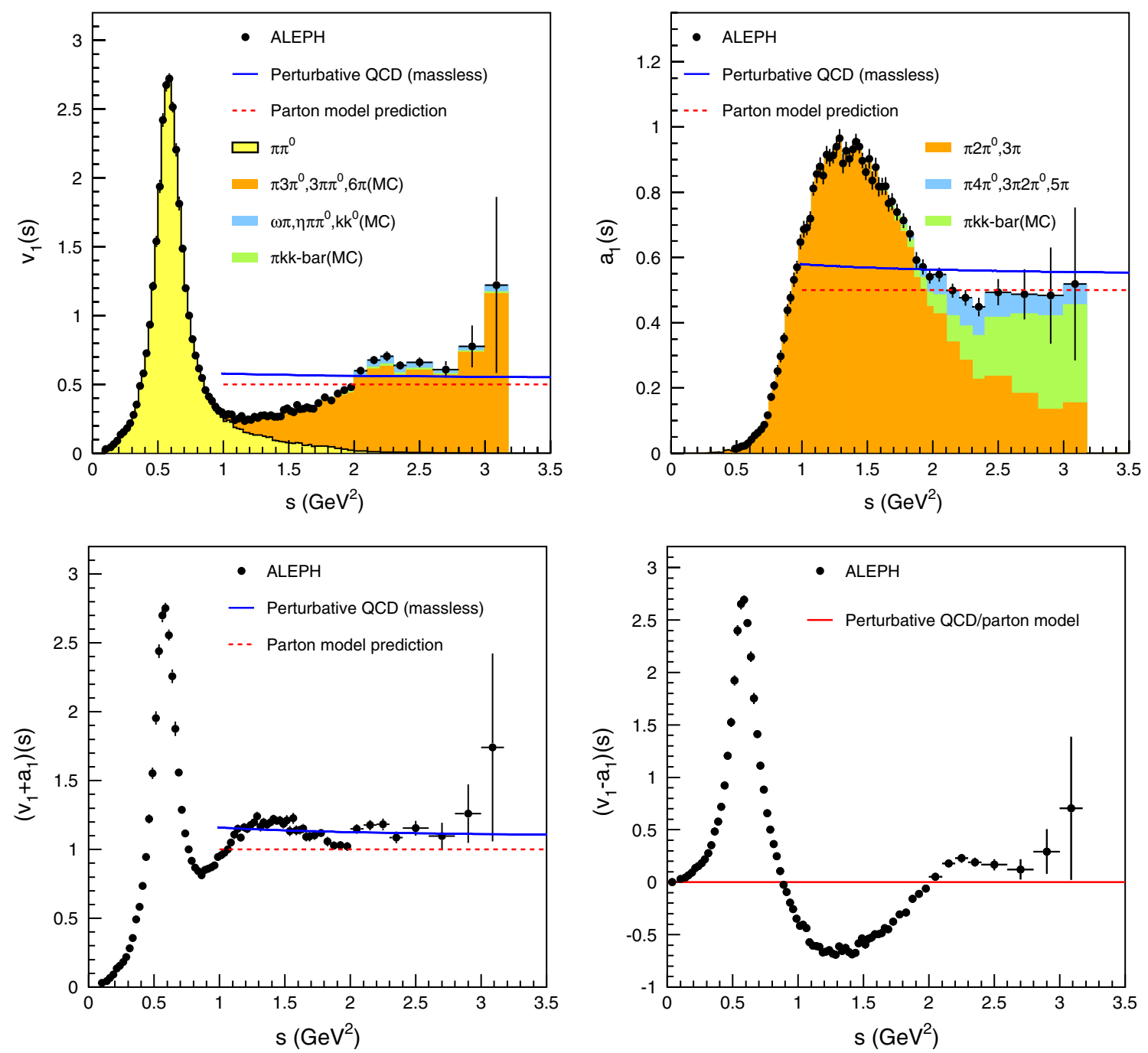

Fig. 5 Updated ALEPH $\tau$ spectral functions. The shaded areas indicate the contributions from the exclusive $\tau$ decay channels, where the shapes of the contributions labelled 'MC' are taken from the MC simulation. The lines show the predictions from the naive parton model and from massless perturbative QCD using $\alpha_{s}\left(M_{Z}^{2}\right)=0.120$, respectively.

with the residual non-logarithmic electroweak correction $\delta_{\mathrm{EW}}^{\prime}=0.0010$, neglected in the following, and the dimension $D=2$ contribution $\delta_{u d, V / A}^{(2-\text { mass })}$ from quark masses which is lower than $0.1 \%$ for $u, d$ quarks. The term $\delta^{(0)}$ is the massless perturbative contribution, while the $\delta^{(D)}$ are the operator product expansion (OPE) terms expressed in powers of $m_{\tau}^{-D}$. In Ref. [3] the perturbative contribution was obtained at third-order in $\alpha_{s}$ while resumming some higherorder contributions using the so-called contour-improved expansion in the complex energy plane. Here we take advantage of a more recent calculation of the fourth-order perturbative coefficient [18], as we had done in a subsequent analysis [17].

The results of the fits to $R_{\tau, V / A / V+A}$ and the normalised moments to the QCD parametrisation are given in Table 4.

Top left: the vector spectral function $V$. Top right the axial-vector spectral function A. Bottom left the $V+A$ spectral function. Bottom right the $V-A$ spectral function. This figure supersedes Figs. 62-65 of [3] and Fig. 2 of [17]

Experimental and theoretical uncertainties are separately given. Since there remains some controversy about the proper choice of the perturbative expansion (fixed-order truncation, FOPT, or contour-improved method, CIPT) the final $\alpha_{s}$ results are given as the average of the two results using the $V+A$ spectral function. A theory uncertainty equal to half their difference is added. The gluon condensate $\left\langle\frac{\alpha_{s}}{\pi} G G\right\rangle$ coming from the $D=4$ contribution is separately treated, the remaining part being calculated from the known quark masses and condensates. Table 5 provides the correlation matrices for the fitted parameters. Agreement is observed between the results in Refs. [3,17] and the ones presented here.

The fit to the $V+A$ data using the FOPT method gives $\alpha_{s}\left(m_{\tau}^{2}\right)=0.324$. Averaging with the CIPT result (see 


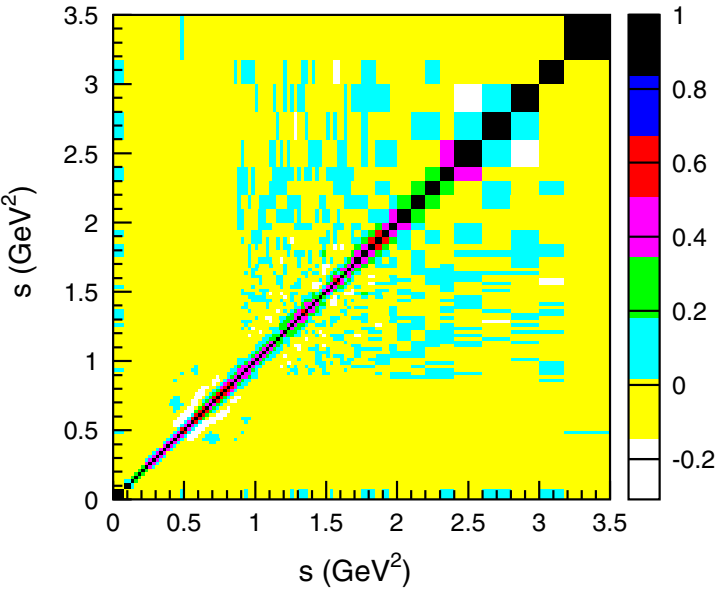

Fig. 6 Display of the correlation matrices for the vector spectral function. Left statistical, with correlations induced by the unfolding. Right statistical and systematic uncertainties combined, with larger correla-

Table 1 Previous and new fit results of the ALEPH pion form factor in the $\tau \rightarrow \pi \pi^{0} v_{\tau}$ channel using the Gounaris-Sakurai (GS) parametrisation. The parameters $m_{\rho^{ \pm}(1700)}$ and $\Gamma_{\rho(1700)}$ are kept fixed to values obtained from 2005 fits of $e^{+} e^{-}$data extending in mass-squared up to $3.6 \mathrm{GeV}^{2}[3]$

\begin{tabular}{lll}
\hline Parameter & ALEPH 2005 & This analysis \\
\hline$m_{\rho^{ \pm}(770)}(\mathrm{MeV})$ & $775.5 \pm 0.7$ & $775.5 \pm 1.1$ \\
$\Gamma_{\rho^{ \pm}(770)}(\mathrm{MeV})$ & $149.0 \pm 1.2$ & $151.4 \pm 1.9$ \\
$\beta$ & $0.120 \pm 0.008$ & $0.120 \pm 0.016$ \\
$\phi_{\beta}($ degrees $)$ & $153 \pm 7$ & $177 \pm 17$ \\
$m_{\rho^{ \pm}(1450)}(\mathrm{MeV})$ & $1328 \pm 15$ & $1404 \pm 29$ \\
$\Gamma_{\rho(1450)}(\mathrm{MeV})$ & $468 \pm 41$ & $474 \pm 84$ \\
$\gamma$ & $0.023 \pm 0.008$ & $0.012 \pm 0.022$ \\
$m_{\rho^{ \pm}(1700)}(\mathrm{MeV})[$ fixed $]$ & 1713 & 1713 \\
$\Gamma_{\rho(1700)}(\mathrm{MeV})[$ fixed $]$ & 235 & 235 \\
$\chi^{2} / \mathrm{DF}$ & $119 / 110$ & $50.4 / 69$ \\
\hline
\end{tabular}

Table 2 Spectral moments of vector $(V)$, axial-vector $(A)$ and vector plus axial-vector $(V+A)$ inclusive $\tau$ decays. The errors give the total experimental uncertainties including statistical and systematic effects. This table supersedes Table 23 of [3] and Table 3 of [17]

\begin{tabular}{lllll}
\hline Moment & $l=0$ & $l=1$ & $l=2$ & $l=3$ \\
\hline$D_{V}^{1 l}$ & 0.71726 & 0.16911 & 0.05313 & 0.02254 \\
$\Delta^{\exp } D_{V}^{1 l}$ & 0.00164 & 0.00042 & 0.00037 & 0.00026 \\
$D_{A}^{1 l}$ & 0.70940 & 0.14885 & 0.06586 & 0.03191 \\
$\Delta^{\exp } D_{A}^{1 l}$ & 0.00211 & 0.00045 & 0.00032 & 0.00027 \\
$D_{V+A}^{1 l}$ & 0.71343 & 0.15924 & 0.05934 & 0.02710 \\
$\Delta^{\exp } D_{V+A}^{1 l}$ & 0.00135 & 0.00029 & 0.00025 & 0.00020 \\
\hline
\end{tabular}

Table 4) and adding to the theoretical uncertainty half the FOPT vs. CIPT difference $( \pm 0.009)$ as explained above, we find for the $V+A$ spectral function

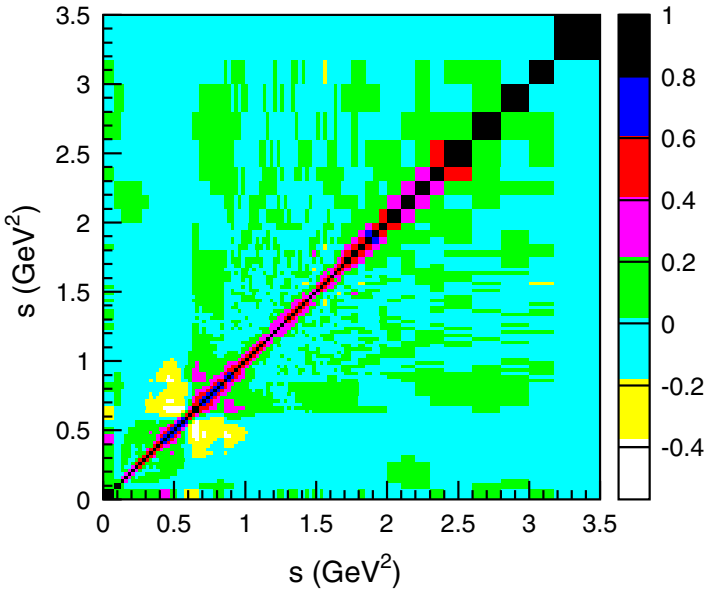

tions and anticorrelations found in the $\rho$ region due to $\pi^{0}$ reconstruction, mass calibration and resolution effects

Table 3 Experimental correlations between the moments $D_{V / A / V+A}^{k l}$. There are no correlations between $R_{\tau, V+A}$ and the corresponding moments. This table supersedes Table 24 of [3] and Table 4 of [17]

\begin{tabular}{llllr}
\hline Vector & $D_{V}^{10}$ & $D_{V}^{11}$ & $D_{V}^{12}$ & \multicolumn{1}{c}{$D_{V}^{13}$} \\
\hline$R_{\tau V}$ & -0.377 & 0.215 & 0.365 & 0.389 \\
$D_{V}^{10}$ & 1 & -0.615 & -0.929 & -0.959 \\
$D_{V}^{11}$ & - & 1 & 0.803 & 0.597 \\
$D_{V}^{12}$ & - & - & 1 & 0.956 \\
\hline Axial-vector & $D_{A}^{10}$ & $D_{A}^{11}$ & $D_{A}^{12}$ & $D_{A}^{13}$ \\
\hline$R_{\tau A}$ & -0.659 & 0.420 & 0.589 & 0.594 \\
$D_{A}^{10}$ & 1 & -0.429 & -0.899 & -0.970 \\
$D_{A}^{11}$ & - & 1 & 0.701 & 0.414 \\
$D_{A}^{12}$ & - & - & 1 & 0.934 \\
\hline$V+A$ & $D_{V+A}^{10}$ & $D_{V+A}^{11}$ & $D_{V+A}^{12}$ & $D_{V+A}^{13}$ \\
\hline$D_{V+A}^{10}$ & 1 & -0.483 & -0.906 & -0.969 \\
$D_{V+A}^{11}$ & - & 1 & 0.743 & 0.508 \\
$D_{V+A}^{12}$ & - & - & 1 & 0.949 \\
\hline
\end{tabular}

$\alpha_{S}\left(m_{\tau}^{2}\right)=0.332 \pm 0.005_{\exp } \pm 0.011_{\text {theo }}$,

which after evolution to $M_{Z}^{2}$ (see Ref. [17] for details) gives

$\alpha_{s}\left(M_{Z}^{2}\right)=0.1199 \pm 0.0006_{\exp } \pm 0.0012_{\text {theo }} \pm 0.0005_{\text {evol }}$

$$
=0.1199 \pm 0.0015_{\text {tot }},
$$

where the third quoted uncertainty is due to the evolution. 
Table 4 Contour-improved (CIPT) fit results of $\alpha_{s}\left(m_{\tau}^{2}\right)$ and the OPE non-perturbative contributions for vector $(V)$, axial-vector $(A)$ and $(V+A)$ combined fits using the corresponding ratio $R_{\tau}$ and the spectral moments as input parameters. Where two errors are given the first is experimental and the second theoretical. The $\delta^{(2)}$ term is the pure theoretical prediction with quark masses varying within their prescribed range (see text). The quark condensates in the $\delta^{(4)}$ term are fixed to their theoretical values within uncertainties and only the gluon condensate is varied as a free parameter. The total non-perturbative contribution is the sum $\delta_{\mathrm{NP}}=\delta^{(4)}+\delta^{(6)}+\delta^{(8)}$. This table supersedes the corresponding results shown in Table 25 of [3] and Table 5 of [17]

\begin{tabular}{llll}
\hline Fitted variable & Vector $(V)$ & Axial-Vector $(A)$ & $V+A$ \\
\hline$\alpha_{s}\left(m_{\tau}^{2}\right)$ & $0.346 \pm 0.007 \pm 0.008$ & $0.335 \pm 0.008 \pm 0.009$ & $0.341 \pm 0.005 \pm 0.006$ \\
$\left\langle\frac{\alpha_{s}}{\pi} G G\right\rangle\left(\mathrm{GeV}^{4}\right)$ & $(-0.5 \pm 0.3) \cdot 10^{-2}$ & $(-3.4 \pm 0.4) \cdot 10^{-2}$ & $(-2.0 \pm 0.3) \cdot 10^{-2}$ \\
$\delta^{(6)}$ & $(2.8 \pm 0.2) \cdot 10^{-2}$ & $(-3.7 \pm 0.2) \cdot 10^{-2}$ & $(-4.6 \pm 1.5) \cdot 10^{-3}$ \\
$\delta^{(8)}$ & $(-8.2 \pm 0.5) \cdot 10^{-3}$ & $(10.9 \pm 0.5) \cdot 10^{-3}$ & $(1.3 \pm 0.3) \cdot 10^{-3}$ \\
\hline$\chi^{2} / 1 \mathrm{DF}$ & 0.43 & 3.4 & 1.1 \\
\hline$\delta^{(2)}$ & $(-3.2 \pm 3.0) \cdot 10^{-4}$ & $(-5.1 \pm 3.0) \cdot 10^{-4}$ & $(-4.2 \pm 2.0) \cdot 10^{-4}$ \\
$\delta^{(4)}$ & $(1.0 \pm 1.6) \cdot 10^{-4}$ & $(-6.3 \pm 0.1) \cdot 10^{-3}$ & $(-3.1 \pm 0.1) \cdot 10^{-3}$ \\
Total $\delta_{\mathrm{NP}}$ & $(2.0 \pm 0.3) \cdot 10^{-2}$ & $(-3.2 \pm 0.2) \cdot 10^{-2}$ & $(-6.4 \pm 1.3) \cdot 10^{-3}$ \\
\hline
\end{tabular}

Table 5 Correlation matrices according to the fits presented in Table 4 for vector (left table), axial-vector (middle) and ( $V+A)$ (right table). As the gluon condensate contributes only insignificantly to $\delta^{(4)}$, the correlations to the total $\delta^{(4)}$ term are small. This table supersedes Table 26 of [3]

\begin{tabular}{|c|c|c|c|c|c|c|c|c|c|}
\hline Moment & $\langle G G\rangle_{V}$ & $\delta_{V}^{(6)}$ & $\delta_{V}^{(8)}$ & $\langle G G\rangle_{A}$ & $\delta_{A}^{(6)}$ & $\delta_{A}^{(8)}$ & $\langle G G\rangle_{V+A}$ & $\delta_{V+A}^{(6)}$ & $\delta_{V+A}^{(8)}$ \\
\hline$\alpha_{s}\left(m_{\tau}^{2}\right)$ & -0.50 & -0.61 & -0.71 & -0.56 & 0.80 & -0.76 & -0.32 & 0.55 & -0.64 \\
\hline$\langle G G\rangle_{V / A / V+A}$ & 1 & 0.44 & 0.71 & 1 & -0.53 & 0.78 & 1 & -0.10 & 0.54 \\
\hline$\delta_{V / A / V+A}^{(6)}$ & - & 1 & 0.92 & - & 1 & -0.92 & - & 1 & -0.87 \\
\hline$\delta_{V / A / V+A}^{(8)}$ & - & - & 1 & - & - & 1 & - & - & 1 \\
\hline
\end{tabular}

\section{Update of the contribution to the muon magnetic anomaly}

The vector spectral functions are useful input to the dispersion relations used to estimate the contribution from hadronic vacuum polarisation to the muon magnetic anomaly. They are complementary to the spectral functions measured in $e^{+} e^{-}$ annihilation, but need to be corrected for isospin-breaking (IB) effects. Here we repeat our analysis [9] using the updated ALEPH results.

In the threshold region below $0.13 \mathrm{GeV}^{2}$ the data are poor, so an expansion constrained at $s=0$ is used [9]. The fits are shown in Fig. 7. Above this value the data are directly integrated, with the results given in Table 6. For comparison the previous ALEPH values (in $10^{-10}$ units) were $9.46 \pm 0.33_{\exp }$ below $0.36 \mathrm{GeV}$ and $499.2 \pm 5.2$ exp between 0.36 and 1.8 $\mathrm{GeV}$, the other uncertainties being identical. So the new values are somewhat higher, especially near threshold, while the experimental uncertainties on the spectral function shape is smaller. The latter change is not a consequence of the new unfolding, but originates from a technical problem in the previous estimate of systematic uncertainties. For the evaluation of the uncertainties affecting the shape of the spectral functions, the normalisation of the invariant mass spectra, given

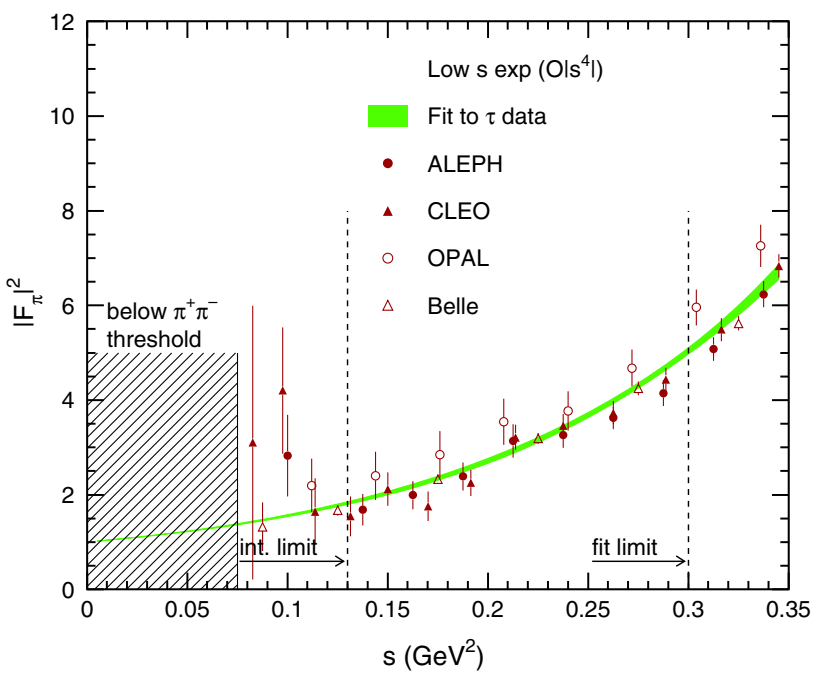

Fig. 7 Fit of the pion form factor from $4 m_{\pi}^{2}$ to $0.3 \mathrm{GeV}^{2}$ using a third order expansion with the constraint $F(0)=1$ and using the measured pion charge radius-squared from space-like data. The result of the fit is integrated only up to $0.13 \mathrm{GeV}^{2}$. This figure supersedes the corresponding plot in Fig. 4 of [9]

by the measured branching fractions, must be kept invariant. This was not enforced in [3] leading to doubly assigned systematic effects. 
Table 6 The isospin-breaking-corrected $a_{\mu}^{\text {had,LO }}[\pi \pi, \tau]$ (in units of $10^{-10}$ ) from the measured mass spectrum by ALEPH, CLEO, OPAL and Belle, and the combined spectrum using the corresponding branching fraction values. The results are shown separately in two different energy ranges. The first errors are due to the shapes of the mass spectra, which also include very small contributions from the $\tau$-mass and $\left|V_{u d}\right|$ uncertainties. The second errors originate from $B_{\pi \pi^{0}}$ and $B_{e}$, and the third errors are due to the isospin-breaking corrections, which are partially anti-correlated between the two energy ranges. The last row gives the evaluations using the combined spectra. This table supersedes the corresponding results shown in Table 2 of [9]

\begin{tabular}{|c|c|c|}
\hline \multirow[t]{2}{*}{ Experiment } & \multicolumn{2}{|l|}{$a_{\mu}^{\mathrm{had}, \mathrm{LO}}[\pi \pi, \tau]\left(10^{-10}\right)$} \\
\hline & $2 m_{\pi^{ \pm}}-0.36 \mathrm{GeV}$ & $0.36-1.8 \mathrm{GeV}$ \\
\hline ALEPH & $9.80 \pm 0.40 \pm 0.05 \pm 0.07$ & $501.2 \pm 4.5 \pm 2.7 \pm 1.9$ \\
\hline CLEO & $9.65 \pm 0.42 \pm 0.17 \pm 0.07$ & $504.5 \pm 5.4 \pm 8.8 \pm 1.9$ \\
\hline OPAL & $11.31 \pm 0.76 \pm 0.15 \pm 0.07$ & $515.6 \pm 9.9 \pm 6.9 \pm 1.9$ \\
\hline Belle & $9.74 \pm 0.28 \pm 0.15 \pm 0.07$ & $503.9 \pm 1.9 \pm 7.8 \pm 1.9$ \\
\hline Combined & $9.82 \pm 0.13 \pm 0.04 \pm 0.07$ & $506.4 \pm 1.9 \pm 2.2 \pm 1.9$ \\
\hline
\end{tabular}

The results for $2 \pi 2 \pi^{0}$ and $4 \pi$ based on linear combinations of $\tau^{-} \rightarrow \pi^{-} 3 \pi^{0} \nu_{\tau}$ and $\tau^{-} \rightarrow 2 \pi^{-} \pi^{+} \pi^{0} \nu_{\tau}$, evaluated up to $1.5 \mathrm{GeV}$, are $14.70 \pm 0.28_{\exp } \pm 1.01_{B} \pm 0.40_{\mathrm{IB}}$ and $7.07 \pm 0.41_{\text {exp }} \pm 0.48_{B} \pm 0.35_{\mathrm{IB}}$, respectively, to be compared to the previous results $14.89 \pm 1.22_{\exp } \pm 1.02_{B} \pm 0.40_{\mathrm{IB}}$ and $6.31 \pm 1.32_{\exp } \pm 0.42_{B} \pm 0.32_{\mathrm{IB}}$. The large difference in the experimental uncertainties stems from the same problem in the evaluation of systematic uncertainties mentioned above for the $\pi \pi^{0}$ mode.

Using the new values for the $\pi \pi^{0}$ mode from threshold to $1.8 \mathrm{GeV}$ combined with the data from CLEO, OPAL, and Belle and $\pi 3 \pi^{0}$ and $3 \pi \pi^{0}$ modes below $1.5 \mathrm{GeV}$, one gets a contribution from $\tau$-only input to $a_{\mu}^{\text {had,LO }}$ of $537.9 \pm 3.1_{\exp +B} \pm 2.0_{\mathrm{IB}}$ to be compared to the previous value $536.4 \pm 3.5_{\exp +B} \pm 2.0_{\mathrm{IB}}$. This increases the $\tau$ - to $e^{+} e^{-}$-based $[19,20]$ difference from $1.8 \sigma$ to $2.2 \sigma$.

\section{Conclusions}

The ALEPH non-strange spectral functions from hadronic $\tau$ decays have been updated using a new method to unfold the measured mass spectra from detector effects. The new method provides a more accurate unfolding and corrects a problem in the correlation matrix of the published spectral functions [3]. The updated spectral functions have been used to repeat the analyses of [3]: a phenomenological fit to the $\pi \pi^{0}$ mass spectrum, a QCD analysis using the vector, axial-vector, and total non-strange spectral functions, and the computation of the hadronic contribution to the anomalous magnetic moment of the muon. The results obtained, although similar in most cases, supersede those reported in Ref. [3].

Acknowledgments We thank the former ALEPH Collaboration for providing the original data used in this re-analysis.
Open Access This article is distributed under the terms of the Creative Commons Attribution License which permits any use, distribution, and reproduction in any medium, provided the original author(s) and the source are credited.

Funded by $\mathrm{SCOAP}^{3}$ / License Version CC BY 4.0.

\section{References}

1. ALEPH Collaboration, Z. Phys. C 76, 15 (1997)

2. ALEPH Collaboration, Eur. J. Phys. C 4, 409 (1998)

3. ALEPH Collaboration, Phys. Rep. 421, 191 (2005). hep-ex/ 0506072

4. D. Boito, Private communication; D. Boito et al., Nucl. Phys. Proc. Suppl. 218, 104 (2011). arXiv:1011.4426

5. B. Malaescu, arXiv:0907.3791; Proceedings to PHYSTAT2011 workshop, CERN-2011/006. arXiv:1106.3107

6. M. Davier, A. Hoecker, Z. Zhang, Rev. Mod. Phys. 78, 1043 (2006). hep-ph/0507078

7. CKM fitter Group, J. Charles et al., Eur. Phys. J. C 41, 1 (2005). hep-ph/0406184

8. A. Hoecker, V. Kartvelishvili, Nucl. Inst. Meth. A 372, 469 (1996). hep-ph/9509307

9. M. Davier et al., Eur. Phys. J C 66, 127 (2010). arXiv:0906.5443

10. CLEO Collaboration, Phys. Rev. D 61, 112002 (2000). hep-ex/ 9910046

11. OPAL Collaboration, Eur. Phys. J. C 7, 571 (1999). hep-ex/ 9808019

12. Belle Collaboration, Phys. Rev. D 78, 072006 (2008). arXiv:0805. 3773

13. BABAR Collaboration, Phys. Rev. Lett. 103, 231801 (2009). arXiv: 0908.3589

14. BABAR Collaboration, Phys. Rev. D 86, 032013 (2012). arXiv: 1205.2228

15. Numerical spectral function files: http://aleph.web.lal.in2p3.fr/tau/ specfun13.html

16. G.J. Gounaris, J.J. Sakurai, Phys. Rev. Lett. 21, 244 (1968)

17. M. Davier, S. Descotes-Genon, A. Hoecker, B. Malaescu, Z. Zhang, Eur. Phys. J C 56, 305 (2008). [arXiv:0803.0979]

18. P.A. Baikov, K.G. Chetyrkin, J.H. Kuhn, Phys. Rev. Lett 101, 012002 (2008). [arXiv:0801.1821]

19. M. Davier, A. Hoecker, B. Malaescu, Z. Zhang, Eur. Phys. J C71, 1515 (2011). [arXiv:1010.4180]

20. K. Hagiwara, R. Liao, A.D. Martin, D. Nomura, T. Teubner, J. Phys. G 38, 085003 (2011). [arXiv:1105.3149] 